\title{
REVIEW
}

\section{A review of clinical effects associated with metabolic syndrome and exercise in prostate cancer patients}

\author{
JL Kiwata ${ }^{1}$, TB Dorff ${ }^{2}$, ET Schroeder ${ }^{1}$, ME Gross ${ }^{3}$ and CM Dieli-Conwright ${ }^{1}$
}

\begin{abstract}
Androgen deprivation therapy (ADT), a primary treatment for locally advanced or metastatic prostate cancer, is associated with the adverse effects on numerous physiologic parameters, including alterations in cardiometabolic variables that overlap with components of the metabolic syndrome (MetS). As MetS is an established risk factor for cardiovascular mortality and treatment for prostate cancer has been associated with the development of MetS, interventions targeting cardiometabolic factors have been investigated in prostate cancer patients to attenuate the detrimental effects of ADT. Much support exists for exercise interventions in improving MetS variables in insulin-resistant adults, but less evidence is available in men with prostate cancer. Regular exercise, when performed at appropriate intensities and volumes, can elicit improvements in ADT-related adverse effects, including MetS, and contributes to the growing body of literature supporting the role of exercise in cancer survivorship. This review (1) discusses the biologic inter-relationship between prostate cancer, ADT and MetS, (2) evaluates the current literature in support of exercise in targeting MetS and (3) describes the physiological mechanisms by which exercise may favorably alter MetS risk factors in prostate cancer patients on ADT.
\end{abstract}

Prostate Cancer and Prostatic Diseases (2016) 19, 323-332; doi:10.1038/pcan.2016.25; published online 28 June 2016

\section{INTRODUCTION}

The 5-year relative survival for men with all stages of prostate cancer is $98.8 \%$ due in large part to recent therapeutic advances. ${ }^{1}$ With improved cancer-specific survival, consideration of treatmentrelated comorbidities has increased substantially. Androgen deprivation therapy (ADT) is one of the primary methods of treating prostate cancer. When used in combination with primary radiation for locally advanced or high-risk localized disease, ADT is associated with improved disease-free and overall survival. ${ }^{2,3}$ However, due to the marked reduction in circulating testosterone, ADT is associated with detrimental changes to body composition, lipid profile and insulin sensitivity. ${ }^{1}$ Such components comprise the cardiometabolic condition known as metabolic syndrome (MetS). MetS is a group of cardiovascular risk factors including hypertension, central adiposity, hypertriglyceridemia, hyperglycemia and low high-density lipoprotein cholesterol (HDL-C) with insulin resistance (IR) serving as the underlying feature. ${ }^{4}$

MetS is an established risk factor for cardiovascular mortality, which contributes to 17.3 million deaths each year in the United States. ${ }^{5}$ It is present in $>50 \%$ of men undergoing long-term ADT, ${ }^{6}$ potentially exposing this population to higher risk of the onset of cardiovascular disease (CVD) and mortality. Furthermore, MetS may contribute to prostate cancer behavior, ${ }^{7}$ suggesting a role for exercise not only in supportive care but potentially as part of cancer control therapy. Components of MetS are linked to an increased risk of prostate cancer and MetS-related biomarkers such as insulin, insulin-like growth factor-1 (IGF-1), leptin and adiponectin are implicated in the involvement of tumorigenesis. ${ }^{7,8}$

Interventions targeting components of MetS would be expected to reduce the risk of cardiovascular comorbidity in cancer survivors and are particularly warranted to attenuate the detrimental cardiometabolic effects of ADT. One such intervention mode, exercise, is well supported in the literature for improving MetS variables in a variety of populations, including individuals with MetS, older adults and patients with type 2 diabetes mellitus. ${ }^{9-11}$ However, this area is largely understudied in men with prostate cancer, thus there is a lack of evidence to define the optimal use of exercise in prostate cancer patients receiving ADT. The purpose of this review is to (1) discuss the biologic inter-relationship between prostate cancer, ADT and MetS, and (2) describe the mechanistic implications for the utilization of exercise to target MetS and subsequent CVD risk.

\section{MetS AND PROSTATE CANCER}

MetS is a cluster of cardiovascular risk factors including hypertension, central adiposity, hypertriglyceridemia, hyperglycemia and low HDL-C with IR serving as the underlying feature. The definition of MetS by the International Diabetes Federation ${ }^{4}$ is shown in Table 1. With IR, normal levels of glucose are insufficient to produce a normal insulin response from fat, muscle and liver cells, often in the presence of central adiposity as a physical manifestation of this state. IR in fat cells causes hydrolysis of stored triglycerides and elevated levels of free fatty acids (FAs). These free FAs are absorbed by the liver resulting in increased triglycerides, low-density lipoprotein cholesterol, and decreased HDL-C. Furthermore, IR causes reduced glucose uptake in the muscle and decreased glucose storage in the liver, leading to the development of hyperglycemia. ${ }^{12}$

${ }^{1}$ Division of Biokinesiology and Physical Therapy, Ostrow School of Dentistry, University of Southern California, Los Angeles, CA, USA; ${ }^{2}$ Norris Comprehensive Cancer Center, Keck School of Medicine, University of Southern California, Los Angeles, CA, USA and ${ }^{3}$ Center for Applied Molecular Medicine, Keck School of Medicine, University of Southern California, Los Angeles, CA, USA. Correspondence: Professor CM Dieli-Conwright, Division of Biokinesiology and Physical Therapy, Ostrow School of Dentistry, University of Southern California, 1540 E Alcazar Street, CHP 155, Los Angeles, CA 90033, USA.

E-mail: cdieli@usc.edu

Received 5 March 2016; revised 19 April 2016; accepted 17 May 2016; published online 28 June 2016 
Table 1. The International Diabetes Federation Definition of Metabolic Syndrome

Central obesity (defined as waist circumference with ethnicity-specific values) plus any two of the following four factors:

Waist circumference (white males)

Elevated fasting plasma glucose

Reduced HDL cholesterol

Elevated blood pressure

Elevated triglycerides $\geqslant 94 \mathrm{~cm}$

$\geqslant 100 \mathrm{mg} \mathrm{dl}^{-1}$, or previously diagnosed type 2 diabetes

$<40 \mathrm{mg} \mathrm{dl}^{-1}$, or specific treatment for this lipid abnormality

Systolic $\geqslant 130$ or diastolic $\geqslant 85 \mathrm{~mm} \mathrm{Hg}$, or treatment of previously diagnosed hypertension $\geqslant 150 \mathrm{mg} \mathrm{dl}^{-1}$, or specific treatment for this lipid abnormality

Abbreviation: HDL, high-density lipoprotein

Various components of MetS including hyperinsulinemia, ${ }^{13}$ hypertension, ${ }^{14}$ central obesity ${ }^{15}$ and dyslipidemia ${ }^{16}$ have been linked to an increased risk of prostate cancer. Specifically, insulin, an integral component in a number of metabolic pathways such as lipogenesis, steriodogenesis and protein synthesis, elicits proliferative and anti-apoptotic actions in many cell types. ${ }^{17,18}$ Insulin stimulates the production of IGF-1, a peptide growth factor, while suppressing the production of IGF-binding proteins (IGFBPs). ${ }^{19}$ As IGFBPs regulate the amount of circulating IGF-1 available to bind to the IGF-1 receptor, higher levels of insulin increase the amount of bioavailable IGF-1, provoking the signaling of growth or survival. In MetS, both hyperglycemia and hyperinsulemia increase bioavailable IGF-1 likely due to the suppression of IGBPs and increases in IGF-1 synthesis, respectively. ${ }^{18}$

Elevated circulating IGF-1 has been epidemiologically linked to risk of various cancer types including prostate cancer, ${ }^{20-22}$ and in vivo models implicate IGF-1 in prostate tumorigenesis. ${ }^{7,8,23}$ In addition, binding of IGF-1 to the IGF-1 receptor activates several pathways, such as the phosphatidylinositol 3-kinase (PI3K)/Akt and the Ras/mitogen-activated protein kinase pathways, that mitigate cell processes including cell growth, survival and angiogenesis (usually upregulated in $30-50 \%$ of prostate cancer) with downstream effects culminating in increased glucose metabolism, inhibition of apoptosis and stimulation of cell proliferation. The $\mathrm{PI} 3 \mathrm{~K} /$ Akt pathway is one of the most commonly altered pathways in epithelial cancers including prostate cancers. ${ }^{24}$ Of importance is the reciprocal feedback regulation in prostate cancer cells between androgen receptor signaling and signaling through the PI3K/Akt/mTOR pathway, which has been implicated in the emergence of androgen-independent tumors. Indeed, IGF-1 is able to stimulate androgen-sensitive and androgen-independent prostate cancer in human cell lines. ${ }^{22}$

MetS is associated with increased serum estradiol levels, decreased sex hormone-binding globulin concentration and decreased free testosterone levels. Higher concentrations of estrogen may promote testosterone-induced carcinogenesis and may be responsible for more aggressive prostate tumors. ${ }^{25,26}$ MetS is associated with the production of various pro-inflammatory cytokines and growth factors, such as leptin and adiponectin, which may lead to genomic instability and a greater risk of cancer development. ${ }^{27}$ In vitro, leptin stimulates the growth of androgeninsensitive prostate cancer cells, whereas adiponectin inhibits angiogenesis. Thus, increased levels of leptin and lower levels of adiponectin contribute to the appearance of more advanced, less differentiated prostate tumors. ${ }^{28}$ Collectively, the aforementioned consequences of MetS on prostate cancer may pose detrimental effects on tumor development and severity, making MetS an important target for attenuation using interventions such as regular exercise.

\section{ADT AND PROSTATE CANCER}

Most patients with localized or regional prostate cancer treated at early stages have favorable outcomes, with a 5 -year specific survival rate near $100 \%{ }^{1}$ Even patients with metastatic prostate cancer anticipate a median survival of 4 years, which has recently increased due to the improved treatment regimens. ADT, which may be attained through gonadotropin releasing hormone analogs (medical castration) or bilateral orchiectomy (surgical castration), is a cornerstone in the treatment of prostate cancer not only for men with metastatic disease but also as an adjunct to curative-intent therapy, particularly in high-risk disease. ${ }^{29-32}$ Benefits of ADT are substantial and include objective tumor regression, extended survival, and relief of urinary symptoms and bone pain. ${ }^{33-35}$

However, a life-threatening health concern related to ADT use is its association with CVD and sudden cardiac death. Although confounded by differing population characteristics, some large observational studies have identified an association between ADT use and increased risk of CVD, myocardial infarction and sudden cardiac death. ${ }^{36-38}$ Thus, consideration of cardiovascular risk factors such as MetS is necessary due to the impact it may have on the development of CVD. The following section will focus on the effect of ADT on MetS as it pertains to cardiometabolic risk in men with prostate cancer on ADT.

\section{MetS AND ADT}

Specific components of MetS resulting from ADT that influence the development of diabetes and CVD include body composition, lipid profiles and IR.

\section{Body composition}

Men with prostate cancer treated with ADT experience symptoms of hypogonadism with the adverse effects including metabolic alterations and unfavorable changes in body composition such as weight gain, loss of muscle mass, increased fat mass and decreased muscle strength. ${ }^{39}$ Because of the suppression of androgen synthesis and signaling, a reduction in lean mass and increase in fat mass, known as sarcopenic obesity, occurs as early as 3-6 months after initiation of ADT. ${ }^{40-42}$ A review of 16 studies reported an average $7.7 \%$ increase in body fat and $2.8 \%$ reduction in lean mass in men with prostate cancer on ADT, with longer duration of treatment associated with greater changes in body composition. ${ }^{41}$ Decreased lean mass is associated with reduced muscle strength and impaired physical performance. $^{43}$

\section{Lipid profiles}

Alterations in lipid profiles are highly variable during ADT use; however, many studies have reported increases in total cholesterol, triglycerides and HDL-C. ${ }^{40,44,45}$ The increase in HDL-C observed while on ADT is a beneficial consequence, although it is unknown whether this improvement provides a cardio-protective effect. Regardless, increases in HDL-C are seen as early as 3-6 months within initiation of treatment. ${ }^{44,45}$ Because of the variability in lipid profile changes following ADT use, further investigation is warranted to determine the overall impact of ADT on cardiometabolic risk in this population. 
Insulin resistance

IR accompanies prediabetes, diabetes and obesity, and is an independent risk factor for CVD. ADT is associated with increased $\mathrm{IR}$, independent of body composition and age. ${ }^{46}$ Compromised insulin sensitivity as a result of ADT occurs as an early consequence, increases in severity over time, and eventually culminates in hyperglycemia and finally type 2 diabetes. ${ }^{37}$ ADT further exacerbates insulin metabolism by increasing fasting insulin levels ${ }^{44,47}$ and decreasing insulin sensitivity. ${ }^{48,49}$ Changes in insulin sensitivity have been noted with short $(12 \text { weeks })^{49}$ or long-term (over 45 months) ${ }^{46}$ ADT use. Likely to exacerbate the observed development of IR are unfavorable changes in body composition, mainly increased visceral adiposity, following ADT use, thus resulting in a cardiometabolic adverse side effect such as MetS. In particular, the adipocytokines leptin and adiponectin, both secreted by adipose tissue, function to modulate insulin sensitivity and are elevated during ADT use. ${ }^{48,50}$ Leptin is involved in appetite and the regulation of energy homeostasis, with levels secreted from adipose tissue in proportion to central adiposity. Circulating levels of leptin increase during ADT use, with or in the absence of increased fat deposition. ${ }^{50}$ The actions of adiponectin generally oppose those of leptin such that reductions in adiponectin levels are observed with obesity, type 2 diabetes, CVD, hypertension and MetS. Adiponectin is normally suppressed by testosterone, ${ }^{51}$ thus the observed increase in adiponectin during ADT is due to the lack of suppression by testosterone. Notably, the effects of adiponectin on insulin sensitivity occurring with ADT use does not overcome the effects of androgen deprivation on the prevalence of hyperinsulinemia. ${ }^{48,50}$

\section{Managing cardiometabolic risk and MetS}

CVD and diabetes are the leading causes of non-cancer deaths in prostate cancer survivors, ${ }^{52}$ thus it is imperative that men using ADT undergo assessments of their cardiometabolic profile before and intermittently during therapy to prevent MetS and additional comorbidities. The American Heart Association, American Cancer Society and American Urological Association have issued guidelines for men receiving ADT, which includes an evaluation of blood pressure, lipids and glucose within 3-6 months of initiating therapy with assessment annually for long-term therapy. ${ }^{53}$ Additional recommendations have been proposed, although not yet adopted, include: (1) maintenance of ideal body weight and waist circumference; (2) smoking cessation; (3) blood pressure control; (4) lipid control; (5) annual screening for diabetes mellitus; and (6) aspirin therapy with established CVD. ${ }^{54}$ Importantly, these recommendations may be mitigated through regular participation in exercise, thus the remainder of this review will focus on implications of exercise to attenuate MetS in prostate cancer survivors on ADT.

\section{EXERCISE AS AN INTERVENTION FOR MetS}

Given the plausible association between prostate cancer, ADT and metabolic dysregulation, interventions targeting components of MetS may reduce the risk of cardiovascular comorbidity in cancer survivors as well as slow disease progression. ${ }^{55}$ Indeed, much support for improving MetS variables through exercise has been demonstrated in a variety of populations, including healthy adults, individuals with MetS, older adults and patients with type 2 diabetes mellitus. ${ }^{9-11}$ Yet, it is important to note that other lifestyle factors, including diet, smoking and alcohol use, may also contribute to MetS. As such, investigations have utilized a combination of exercise, diet and behavior counseling in their interventions, with the result of reducing MetS prevalence. ${ }^{11}$ As this review focuses on exercise as an intervention for MetS in prostate cancer survivors, the following sections will focus solely on the effects of exercise-rather than multiple lifestyle modifications-in improving MetS, the mechanisms underlying exercise-induced adaptations and the application of evidencebased exercise to survivorship care for patients on ADT.

\section{Exercise types}

Of the exercise interventions, aerobic exercise possesses the greatest amount of support in improving cardiometabolic outcomes in individuals with MetS, albeit none were conducted in cancer survivors. ${ }^{56-60}$ Only two of six studies reported significant reductions in MetS prevalence, whereas all six reported improvements in individual MetS components. ${ }^{56-60}$ The HEalth, Rlsk factors, exercise Training And GEnetics (HERITAGE) Family Study ${ }^{60}$ investigated the efficacy of 20 weeks of supervised aerobic training (AT) consisting of 3 days per week at 55\% maximal oxygen uptake $\left(\mathrm{VO}_{2 \max }\right)$ for $30 \mathrm{~min}$ and progressing to $75 \%$ $\mathrm{VO}_{2 \max }$ for $50 \mathrm{~min}$. In their sample of 621 participants, $16.9 \%$ $(105 / 621)$ met the ATP III criteria for MetS at baseline, and following the intervention, $30.5 \%$ (32/105) were no longer classified as having MetS. Similarly, the Studies of a Targeted Risk Reduction Intervention through Defined Exercise (STRRIDE) reported significant reductions in MetS prevalence in the exercise groups compared with a control group, ${ }^{59}$ with a low amount of moderate intensity AT ( 19 km per week walking) or a high amount of vigorous intensity AT ( $32 \mathrm{~km}$ per week jogging) improving MetS. In terms of individual MetS components, a reduction in waist circumference appears to be the most common improvement elicited by $\mathrm{AT}^{56-60}$ whereas decreases in blood pressure, ${ }^{57-60}$ decreases in triglycerides ${ }^{59-61}$ and increases in HDL$C$ level ${ }^{56,58-60}$ are observed to a lesser extent.

Resistance exercise training (RT) has been utilized in MetS interventions, but primarily in combination with or compared with AT. A combined intervention of AT+RT appears to be at least as effective as AT alone in improving select MetS criteria. ${ }^{56,61}$ However, in a sample of 86 adults, 8 months of RT, when compared with AT or AT+RT of the same duration, did not change the MetS $z$-score (a continuous score of the five MetS variables), whereas the MetS score was significantly reduced by AT and AT+RT.61

High-intensity interval training (HIT) has recently emerged as an effective approach to improving MetS criteria, $57,62,63$ and in one investigation, ${ }^{57}$ was superior to AT in eliciting improvements. HIT is characterized by bouts of short, vigorous activity interspersed by rest or intervals of low-intensity activity. ${ }^{64}$ Although vigorous activity could consist of either aerobic or resistance exercise, aerobic exercise has traditionally been the modality of choice in clinical populations. In a sample of 32 MetS patients, Tjonna et al. ${ }^{57}$ utilized four 4-min intervals of treadmill running at $90 \%$ heart rate max separated by 3-min active recovery intervals at $70 \%$ heart rate max and observed the removal of four out of five MetS risk factors. In comparison, patients who performed continuous aerobic exercise matched for the same training volume demonstrated improvements in only two MetS variables. In a different study using the same protocol, HIT was found to be no more effective at improving MetS criteria than RT or a HIT+RT protocol in a sample of 43 middle-aged adults with MetS. ${ }^{62}$ These findings suggest that HIT may be a promising alternative to commonly employed AT interventions, with the distinct advantages of having greater time efficiency and being more versatile, as either RT or AT may serve as the vigorous activity of choice. However, future work is needed to address the efficacy of RT, particularly in relation to $\mathrm{HIT}$, in eliciting improvements in MetS variables.

\section{EXERCISE IN PROSTATE CANCER PATIENTS ON ADT}

Although strong evidence exists for the efficacy of exercise training on cardiometabolic risk factors in individuals with MetS, there is less support for the impact of exercise on MetS outcomes in prostate cancer survivors. Numerous randomized controlled 
trials (RCTs) and uncontrolled trials (no randomization or control group) have been conducted on the benefits of exercise during ADT, although primary end points are commonly body composition or physical performance. The few RCTs that have examined the effect of exercise on cardiometabolic outcomes have generally focused on a single risk factor rather than the constellation of factors comprising MetS. Among those studies expressly targeting MetS criteria in prostate cancer survivors, none have reported reduced prevalence of MetS as a result of exercise. In total, eight RCTs examining at least one MetS component in prostate cancer survivors on ADT were found (Table 2) using an electronic search on peer-reviewed articles published between January 1990 and March 2016. ${ }^{65-72}$ Studies were included if the trial was randomized and controlled, participants were prostate cancer patients on ADT, an exercise only intervention was performed rather than a combined intervention of exercise and diet or a self-report of physical activity behavior, and at least one MetS component served as a study end point.

Although the exercise modalities utilized by the eight studies have included AT+RT, AT, RT and HIT, only programs of AT+RT and HIT yielded changes in MetS variables. ${ }^{67,68,70}$ Culos-Reed et al. ${ }^{68}$ reported a significant difference in waist circumference between exercise and control groups following 16 weeks of an AT+RT intervention. Significant decreases in blood pressure were also observed, but only as a within-group difference in the exercise group following the intervention. Similarly, Cormie et al. ${ }^{67}$ observed a significant within-group increase in HDL-C in the exercise group after 12 weeks of combined AT+RT, but with no changes in blood pressure, fasting glucose or triglyceride levels. No significant between-group differences were observed for any variable when the AT+RT group was compared with the control group. Notably, in the first HIT intervention conducted in prostate cancer survivors on ADT, reductions in fasting glucose levels and increases in $\mathrm{HDL}-\mathrm{C}$ were observed following 12 weeks of training; however, no between-group differences in MetS components were found between the exercise group and healthy control subjects. $^{70}$

As nearly all eight interventions met the American Cancer Society's physical activity guidelines of at least $150 \mathrm{~min}$ per week of moderate intensity physical activity or at least 75 min per week of vigorous intensity physical activity, ${ }^{73}$ other factors, such as a higher weekly training volume or greater intensity of exercise, likely contributed to the greater efficacy of the above-mentioned exercise interventions. Culos-Reed et al. ${ }^{68}$ employed up to 5 days per week of moderate intensity home-based AT+RT, whereas Cormie et al. ${ }^{67}$ utilized a supervised program of progressive RT and moderate-to-vigorous intensity AT 2 days per week. Conversely, Hvid et al. ${ }^{70}$ used supervised AT, but employed HIT for 35 min each session, 3 days per week, at intensities ranging between 50 and $100 \%$ maximal oxygen uptake $\left(\mathrm{VO}_{2 \text { max }}\right)$. Therefore, it appears that home-based exercise may be as effective as supervised exercise in altering cardiometabolic variables in prostate cancer survivors provided training volume is high (that is, $>150$ min per week). In addition, shorter duration aerobic exercise can be as effective as longer duration aerobic exercise provided intensity is high (that is, $100 \% \mathrm{VO}_{2 \max }$ ). Finally, it is important to note that factors relating to patient status, such as ADT duration, comorbid CVD or pharmacotherapy (that is, metformin), could potentially alter the efficacy of exercise interventions on cardiometabolic variables. Thus, to ascertain the optimal exercise prescription, future studies should adjust for these intervening factors through stratification or eligibility criteria.

Our laboratory is currently investigating the effects of a 12-week progressive RT intervention in prostate cancer patients on ADT (NCT01909440). ${ }^{74}$ In this RCT, the main inclusion criteria are serum testosterone concentration $<50 \mathrm{ng} \mathrm{dl}^{-1}$ for study duration due to current or previous ADT, completion of radiation therapy
$>4$ weeks before study enrollment, and recovery from major surgery within 6 months. Study end points are comprised of alterations in cardiometabolic variables including all MetS risk factors, body composition, physical function and quality of life. Participants randomized to the exercise group complete three sessions per week of supervised, whole-body RT using a model of systematic progression previously unemployed in exercise interventions for prostate cancer patients on ADT. The progression model, known as periodization, has been used primarily in athletic populations and is regarded as a superior method for optimally eliciting physiological and performance-related improvements. ${ }^{75}$ Periodized RT may serve as an ideal strategy for concomitantly increasing lean body mass while decreasing the fat mass, results not demonstrated with an AT-only intervention, thus optimizing beneficial changes in cardiometabolic variables. For this reason, $\mathrm{RT}$, rather than AT, is utilized in our trial. The attention-control group receives a home-based stretching program and is offered the intervention following study completion. Findings from this study may provide an evidence-based exercise prescription for improving ADT-related adverse effects, including MetS, and contribute to the growing body of literature supporting the role of exercise in cancer survivorship. We are aware of other prospective studies involving exercise and prostate cancer survivorship with several studies incorporating novel ways to deliver exercise prescription, including recreational football-based training (NCT02430792), ${ }^{76}$ Xbox Kinect-based training (NCT01762241), ${ }^{77}$ supervised group AT+RT training (NCT02631681), ${ }^{78}$ or homebased training (NCT02248350). ${ }^{79}$

\section{POTENTIAL MECHANISMS OF EXERCISE-INDUCED CARDIOMETABOLIC IMPROVEMENTS IN PROSTATE CANCER PATIENTS ON ADT}

The relationship between MetS and prostate cancer centers on the role of insulin, IGF-1 and their receptors as key elements in downstream signaling pathways that promote tumor growth. ${ }^{21}$ Previous reviews on the molecular aspects of exercise-induced cardiometabolic improvements have focused on $\mathrm{IR}_{1}^{9,11,80,81}$ with exercise adaptations working to improve insulin sensitivity through the following mechanisms: increasing glucose tolerance, ${ }^{9,11}$ increasing lipid oxidation, ${ }^{9,81}$ and improving immune, metabolic and hormonal factors. ${ }^{80,82}$ This section will briefly review the general mechanisms by which exercise may improve insulin action and favorably alter MetS risk factors in prostate cancer survivors on ADT.

\section{Glucose tolerance}

Most support for exercise-induced improvements in insulin sensitivity arises from mechanistic studies in skeletal muscle investigating insulin signaling in response to AT. Enhanced glucose uptake in the muscle after exercise appears to be mediated by post-receptor insulin signaling and driven by glycogen repletion. ${ }^{9,11}$ One signaling protein that has been studied in detail in response to exercise is glucose transporter isoform 4 (GLUT4), the major insulin-responsive glucose transporter that is highly expressed in the muscle as well as adipose tissue. ${ }^{11}$ In individuals with impaired glucose tolerance, AT has been found to increase GLUT4 protein concentration, ${ }^{83,84}$ as well as lower blood glucose levels, ${ }^{84}$ increase glycogen synthase activity ${ }^{83}$ and increase glucose storage ${ }^{83,84}$ However, the activity of signaling proteins upstream of GLUT4 was not observed to increase after exercise training in both insulin-resistant and type 2 diabetic men. ${ }^{83}$ Furthermore, HIT has been shown to substantially increase GLUT4 protein content $(\sim 369 \%)$ and lower blood glucose levels in type 2 diabetes patients, even with a weekly training volume lower than the American Cancer Society recommendation. ${ }^{85}$ Studies investigating the mechanisms of skeletal muscle adaptation to 


\begin{tabular}{|c|c|c|c|c|c|c|c|c|}
\hline Study & Year & Sample & $\begin{array}{l}\text { Age (years) } \\
\text { (mean } \pm \text { s.d.) }\end{array}$ & Treatment & $\begin{array}{c}\text { Study } \\
\text { duration }\end{array}$ & Exercise intervention & $\begin{array}{c}\text { Frequency } \\
\text { (days per week) }\end{array}$ & Key MetS findings \\
\hline $\begin{array}{l}\text { Bourke } \\
\text { et al. }\end{array}$ & 2014 & $\begin{array}{l}\text { Exe }(n=50) \\
\text { Con }(n=50)\end{array}$ & $\begin{array}{c}\text { Exe } \\
(71.0 \pm 6.0) \\
\text { Con }(71.0 \pm 8.0)\end{array}$ & $\begin{array}{l}A D T \geqslant 6 \text { months and expected to } \\
\text { receive } A D T \text { for study duration }\end{array}$ & 12 weeks & $\begin{array}{l}\text { Small-group supervision of PRT and PAT including } \\
\text { lifestyle advice seminars } \\
\text { PRT: } 2-4 \text { sets of exercises at } 8-12 \text { reps starting at } \\
60 \% \text { RM } \\
\text { PAT: } 30 \text { min at } 55-75 \% \text { HRmax and RPE } 11-13\end{array}$ & 3 & $\begin{array}{l}\text { Exe vs control } \\
\text { BP } \leftrightarrow\end{array}$ \\
\hline $\begin{array}{l}\text { Bourke } \\
\text { et al. }\end{array}$ & 2011 & $\begin{array}{l}\text { Exe }(n=25) \\
\text { Con }(n=25)\end{array}$ & $\begin{array}{c}\text { Exe } \\
(71.3 \pm 6.4) \\
\text { Con }(72.2 \pm 7.7)\end{array}$ & $A D T \geqslant 6$ months & 12 weeks & $\begin{array}{l}\text { Home-based and group supervision of RT and AT } \\
\text { including dietary advice seminars } \\
\text { RT: } 2-4 \text { sets of exercises incorporating large muscle } \\
\text { groups at light-to-moderate intensity } \\
\text { AT: } 30 \text { min at } 55-85 \% \text { HRmax and RPE } 11-15\end{array}$ & $\begin{array}{l}\text { Up to } 5 \\
\text { encouraged }\end{array}$ & $\begin{array}{l}\text { Exe vs control } \\
\text { BP, waist to hip } \\
\leftrightarrow\end{array}$ \\
\hline $\begin{array}{l}\text { Cormie } \\
\text { et } a l^{67}\end{array}$ & 2015 & $\begin{array}{l}\text { Exe }(n=32) \\
\text { Con }(n=31)\end{array}$ & $\begin{array}{l}\text { Exe }(69.6 \pm 6.5) \\
\text { Con }(67.1 \pm 7.5)\end{array}$ & $\begin{array}{l}\text { No prior ADT and expected to } \\
\text { receive ADT for } 3 \text { months }\end{array}$ & 12 weeks & $\begin{array}{l}\text { Small-group supervision of PRT and PAT } \\
\text { PRT: } 1-4 \text { sets of } 8 \text { exercises at } 6-12 \text { RM } \\
\text { incorporating major muscle groups } \\
\text { PAT: } 20-30 \text { min at } 70-85 \% \text { HRmax }\end{array}$ & 2 & $\begin{array}{l}\text { Exe vs control } \\
\text { BP, glucose, } \\
\text { HDL, TG } \leftrightarrow \\
\text { Pre-to-post Exe } \\
\text { HDL } \uparrow \\
\text { BP, glucose, TG } \\
\leftrightarrow\end{array}$ \\
\hline $\begin{array}{l}\text { Culos-Reed } \\
\text { et al. }{ }^{68}\end{array}$ & 2010 & $\begin{array}{l}\text { Exe }(n=53) \\
\text { Con }(n=47)\end{array}$ & $\begin{array}{l}\text { Exe }(67.2 \pm 8.8) \\
\text { Con }(68.0 \pm 8.4)\end{array}$ & $\begin{array}{l}\text { Expected to receive ADT for at } \\
\text { least } 6 \text { months }\end{array}$ & 16 weeks & $\begin{array}{l}\text { Home-based and weekly group sessions consisting } \\
\text { of walking, stretching and Theraband exercises } \\
\text { at a moderate intensity }\end{array}$ & $\begin{array}{c}3-5 \\
\text { encouraged }\end{array}$ & $\begin{array}{l}\text { Exe vs control } \\
\text { BP } \leftrightarrow \\
\text { WC } \downarrow \\
\text { Pre-to-post Exe } \\
\text { BP } \downarrow \\
\text { WC } \leftrightarrow\end{array}$ \\
\hline $\begin{array}{l}\text { Galvao } \\
\text { et al. }\end{array}$ & 2010 & $\begin{array}{l}\text { Exe }(n=29) \\
\text { Con }(n=28)\end{array}$ & $\begin{array}{l}\text { Exe }(69.5 \pm 7.3) \\
\text { Con }(70.1 \pm 7.3)\end{array}$ & $\begin{array}{l}\text { ADT } \geq 2 \text { months and expected } \\
\text { to receive ADT for } 6 \text { months }\end{array}$ & 12 weeks & $\begin{array}{l}\text { Supervised PRT and PAT } \\
\text { PRT: } 2-4 \text { sets of } 8 \text { exercises incorporating major } \\
\text { muscle groups at } 12-6 \text { RM } \\
\text { PAT: } 15-20 \text { min at } 65-80 \% \text { HRmax and RPE } 11-13\end{array}$ & 2 & $\begin{array}{l}\text { Exe vs control } \\
\text { Glucose, HDL, } \\
\text { TG } \leftrightarrow\end{array}$ \\
\hline Hvid et al. ${ }^{70}$ & 2011 & $\begin{array}{l}\text { Exe (ADT; } n=9 \text { ) } \\
\text { Con (healthy; } \\
\quad n=10)\end{array}$ & $\begin{array}{l}\text { Exe }(67.8 \pm 6.4) \\
\text { Con }(68.5 \pm 3.5)\end{array}$ & $A D T \geqslant 3$ months & 12 weeks & $\begin{array}{l}\text { Supervised HIT: } 35 \text { min interval training } 65-100 \% \\
\text { VO }_{2 \max }\end{array}$ & 3 & $\begin{array}{l}\text { Exe vs control } \\
\text { HDL, glucose, } \\
\text { TG } \leftrightarrow \\
\text { Pre-to-post Exe } \\
\text { HDL } \uparrow \\
\text { Glucose } \downarrow \\
\text { TG } \leftrightarrow\end{array}$ \\
\hline Segal et al. ${ }^{72}$ & 2003 & $\begin{array}{l}\text { Exe }(n=82) \\
\text { Con }(n=73)\end{array}$ & $\begin{array}{l}\text { Exe }(68.2 \pm 7.9) \\
\text { Con }(67.7 \pm 7.5)\end{array}$ & ADT for study duration & 12 weeks & $\begin{array}{l}\text { Supervised PRT } \\
\text { PRT: } 2 \text { sets of } 9 \text { exercises incorporating major muscle } \\
\text { groups starting at } 60-70 \% \text { RM. }\end{array}$ & 3 & $\begin{array}{l}\text { Exe vs control } \\
\text { WC } \leftrightarrow\end{array}$ \\
\hline $\begin{array}{l}\text { Santa Mina } \\
\text { et al. }\end{array}$ & 2013 & $\begin{array}{l}\text { AT }(n=13) \\
\text { RT }(n=13)\end{array}$ & $\begin{array}{l}\text { AT }(70.6 \pm 8.1) \\
\text { RT }(73.6 \pm 8.8)\end{array}$ & ADT for study duration & 6 months & $\begin{array}{l}\text { Home-based RT or AT } \\
\text { RT: } 10 \text { exercises incorporating major muscle groups } \\
\text { AT: } 60 \text { min at } 60-80 \% \text { HRmax }\end{array}$ & $\begin{array}{l}\text { Up to } 5 \\
\text { encouraged }\end{array}$ & $\begin{array}{l}\text { AT vs RT } \\
\text { WC } \leftrightarrow\end{array}$ \\
\hline
\end{tabular}


HIT training have centered on the influence of peroxisomeproliferator activated receptor $\gamma$ coactivator $(P G C-1 a)$, regarded as a major regulator of exercise-induced mitochondrial biogenesis and substrate utilization. ${ }^{86}$ Elevated expression of PGC-1a in the skeletal muscle following exercise has been shown to increase glucose uptake, GLUT4 expression and glycogen stores in a transgenic mouse model, whereas PGC-1 a knockout mice exhibit delayed replenishment of glycogen stores after exercise. ${ }^{86}$ Although data from a meta-analysis of six prospective cohorts $(n=274125)$ did not identify a significant association between risk of fatal prostate cancer and impaired glucose tolerance, ${ }^{87}$ men with type 2 diabetes are more likely to present with high-grade prostate tumors, ${ }^{88}$ and exercise is an established method for reducing risk of type 2 diabetes.

\section{Lipid oxidation}

Strong evidence exists for the role of consistent exercise in improving blood lipid profile through decreases in triglyceride and low-density lipoprotein levels, and increases in HDL levels. ${ }^{89}$ As IR has been linked to imbalances in lipid accumulation and oxidation in the skeletal muscle, exercise that alters this mismatch through changes in FA uptake or FA oxidation can significantly improve insulin sensitivity. ${ }^{89}$ These improvements are primarily mediated through changes in muscle substrate utilization that increase FA oxidation at rest and during submaximal exercise. ${ }^{89}$ An increased reliance on FA oxidation was observed at rest ${ }^{90}$ and during lowintensity aerobic exercise in insulin-resistant men after exercise training. ${ }^{91}$ Furthermore, oxidative enzymes responsible for FA oxidation were increased in obese middle-aged adults after a 4month training program, and were accompanied by increases in insulin sensitivity. ${ }^{92}$ However, some authors have suggested that the reduced availability of plasma free FA, rather than FA uptake, may be responsible for improvements in insulin sensitivity. ${ }^{90}$ The availability of free FA may also be important in the context of prostate cancer as observational and preclinical data support FA availability to tumors leads to prostate cancer growth. ${ }^{93,94}$ This is especially relevant in the setting of MetS, as altered blood lipid profiles increase FA availability, potentially fueling prostate tumors with energetic or biosynthetic substrates. ${ }^{95}$ Although the precise impact of exercise-induced increases in lipid oxidation on prostate cancer progression is not yet known, one may speculate that improvements in IR via reduced availability of FA may limit the ability of cancer cells to utilize exogenous free FA in their metabolic requirements.

\section{Immunometabolism}

Dysregulation of the complex interaction between immune and metabolic factors due to excessive adiposity has been implicated in the pathogenesis of several chronic diseases, including type 2 diabetes, CVD and prostate cancer. ${ }^{96,97}$ In particular, excess adipose tissue leads to the elevated production of proinflammatory adipokines, including leptin, tumor necrosis factor and interleukin-6 (IL-6), contributing to a persistent state of lowgrade inflammation. ${ }^{96}$ Regular exercise has been proposed to counter this systemic inflammation through reductions in visceral fat and the subsequent decline in pro-inflammatory adipokines, as well as through the transient rise in anti-inflammatory myokines, or cytokines that are released from contracting the skeletal muscle during exercise. ${ }^{80} \mathrm{IL}-6$ has been identified as the primary myokine mediating the immunological and metabolic response to exercise, despite its pathogenic role in the development of MetS and other chronic disease. ${ }^{98}$ In particular, although high resting plasma levels of IL-6 are associated with MetS, AT has been shown to decrease resting plasma IL- 6 levels and increase expression of its receptor, IL-6ra, such that sensitivity to IL- 6 is increased in the skeletal muscle. ${ }^{99}$ These observations have led one group of authors to hypothesize that physical inactivity leads to elevated plasma levels of IL- 6 and therefore IL- 6 resistance, which parallels elevated plasma levels of insulin and IR. ${ }^{82}$ This hypothesis is supported by evidence demonstrating the beneficial effect of IL- 6 on insulin sensitivity. Specifically, infusion of IL- 6 has been shown to increase glucose uptake and translocation of GLUT4 in rat $\mathrm{L} 6$ muscle myotubes ${ }^{100}$ and enhance whole-body insulin sensitivity during a hyperinsulinemic-euglycemic clamp in healthy humans. $^{100}$ In addition, elevations in the anti-inflammatory cytokine IL-10 following exercise, attributed to contractionmediated rises in IL-6, are purported to increase insulin sensitivity, ${ }^{80}$ as treatment with $\mathrm{IL}-10$ was demonstrated to attenuate IR following acute lipid infusion in mice. ${ }^{101}$ Collectively, these findings suggest that IL-6 released from contracting muscle modulates positive metabolic and immune responses that work to improve IR, and possibly MetS. The dynamic changes in IL-6 due to exercise may also serve a beneficial role in tumorgenesis, as consistent aerobic exercise has been shown to decrease tumor growth through intratumoral infiltration by IL-6-sensitive natural killer cells. ${ }^{102}$ Furthermore, aerobic exercise favorably altered metastatic gene expression while decreasing resting levels of IL-6 in an orthotopic model of murine prostate cancer, suggesting reductions in resting levels of IL- 6 may be related to tumor regression to a less aggressive phenotype. ${ }^{103}$ Certainly, further research is needed to elucidate exercise-induced changes in inflammation and its relation to prostate cancer progression.

\section{IGF-1:IGFBP}

Epidemiological studies have demonstrated a significant association between high IGF-1 levels, low IGFBP levels and increased risk of prostate cancer, ${ }^{21}$ spurring investigations into the effects of exercise on prostate tumor growth and cancer progression. Both insulin and IGF-1 have been shown to suppress the production of sex hormone-binding globulin, resulting in increased levels of free testosterone that may stimulate prostate tumor cell growth. ${ }^{104}$ Several investigations have provided support for alteration of IGF axis factors through acute ${ }^{105}$ and chronic exercise training. ${ }^{106-109}$ In particular, serum from healthy men who performed a single bout of exercise ${ }^{105}$ or regular aerobic exercise for at least 5 days $^{106-109}$ was found to reduce proliferation and increase apoptosis of serum-stimulated lymph node cancer of the prostate tumor cells in vitro. Furthermore, the exercise training reduced serum insulin and IGF-1 levels, and increased IGFBP-1 and sex hormone-binding globulin levels. When IGFBP-1 was added back to the exercise serum, lymph node cancer of the prostate growth was reduced and apoptosis was increased. ${ }^{109}$ Although serum in these aforementioned experiments was obtained from healthy males, a recent study expanded these methods to prostate cancer. ${ }^{71}$ A sample of 13 prostate cancer survivors on ADT performed 6 months of RT, which was found to significantly increase serum IGFBP-3 and reduce IGF-1:IGFBP-3 ratio in comparison with a control group. Although it appears that chronic RT beneficially alters factors in the IGF axis to reduce bioavailable IGF-1, larger RCTs are needed to ascertain the clinical impact of these results on the progression of prostate cancer.

\section{CONCLUSION}

Currently, there is insufficient evidence to directly indicate specific exercise guidelines to ameliorate MetS risk factors in prostate cancer patients on ADT. As ADT results in severe hypogonadism, and hypogonadism is an independent risk factor for MetS, ADTinduced metabolic complications are a significant issue in survivorship care (Figure 1). Furthermore, MetS is associated with worse prognosis in prostate cancer patients, including higher tumor grade, recurrence and increased mortality. ${ }^{55}$ Yet, a substantial body of work exists in support of exercise as a 


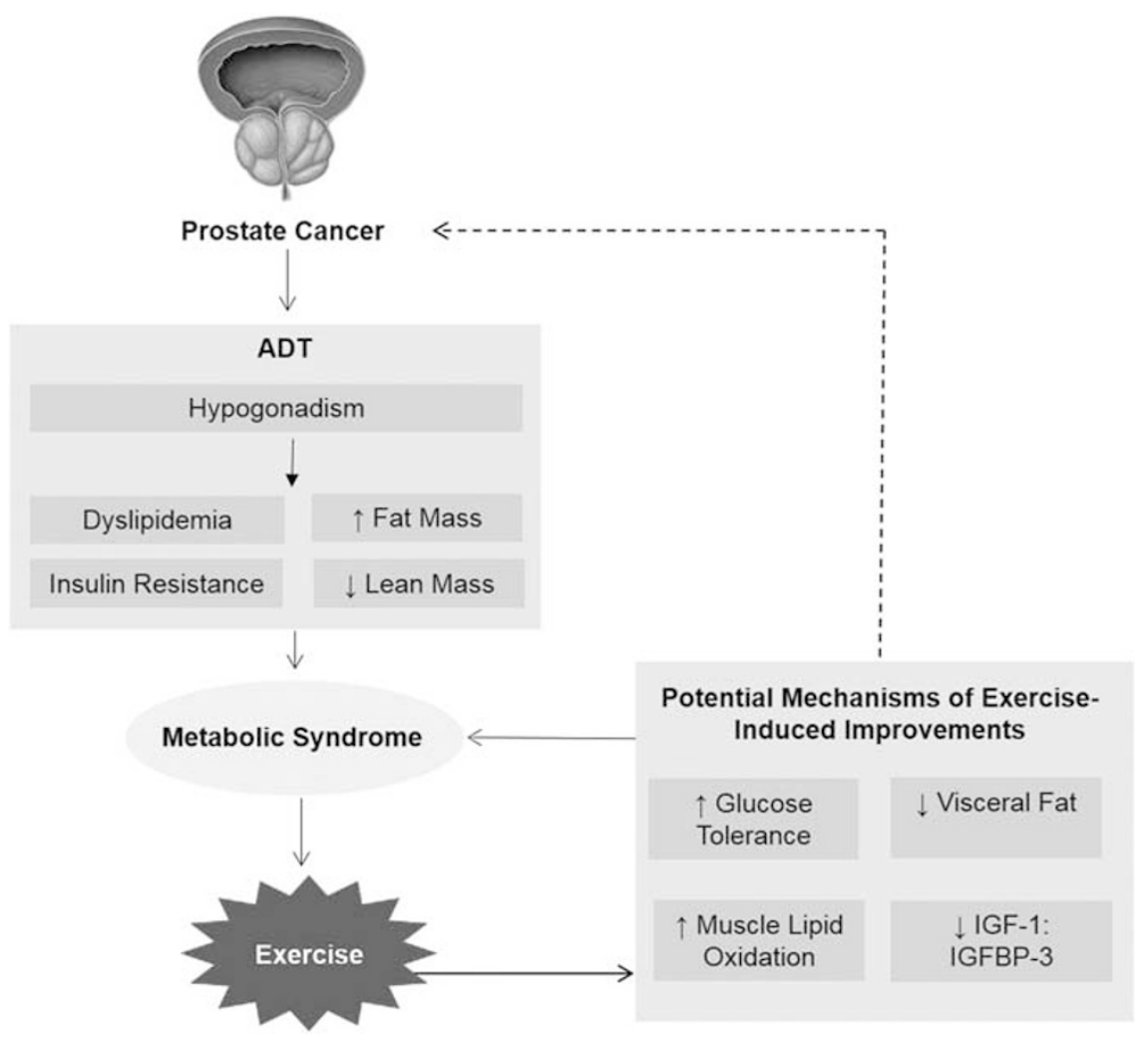

Figure 1. Relationship between prostate cancer, androgen deprivation therapy (ADT), metabolic syndrome and potential mechanisms of improvement due to exercise. IGF, insulin-like growth factor; IGFBP, IGF-binding protein.

Table 3. Summary of evidence-based exercise recommendations for improving MetS variables in prostate cancer patients on ADT

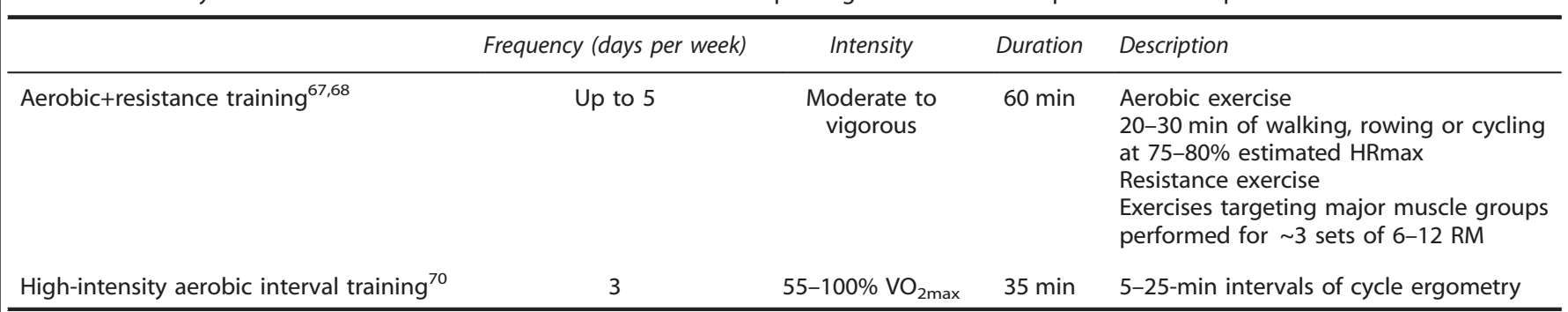

Abbreviations: ADT, androgen deprivation therapy; HRmax, heart rate max; MetS, metabolic syndrome; $\mathrm{RM}$, repetition maximum; $\mathrm{VO}_{2 \max }$ maximal oxygen uptake.

therapeutic intervention for favorably altering MetS variables, albeit in populations other than cancer survivors. In addition, exercise interventions conducted in prostate cancer survivors on ADT suggest that various forms of exercise programs, including resistance, aerobic and high-intensity interval training, are safe and well tolerated by patients, and may beneficially modify cardiometabolic risk factors. Thus, the features of interventions that elicit positive changes in MetS variables can be summarized to outline a possible exercise prescription (Table 3). Although large-scale RCTs are needed to ascertain the precise exercise prescription necessary to improve MetS factors arising from ADT administration, patients can still benefit from consistent exercise as improvements in muscle mass, strength, physical function and psychological well-being have been demonstrated. ${ }^{68,69,72}$ Therefore, participation in an appropriately designed exercise program may confer additional health benefits and should be encouraged for prostate cancer survivors receiving ADT.

\section{CONFLICT OF INTEREST}

The authors declare no conflict of interest.

\section{ACKNOWLEDGEMENTS}

Dr Dorff is supported by P30CA014089 from the National Cancer Institute.

\section{REFERENCES}

1 Saylor PJ, Smith MR. Metabolic complications of androgen deprivation therapy for prostate cancer. J Urol 2013; 189: S34-S42.

2 Bolla M, Gonzalez D, Warde P, Dubois JB, Mirimanoff RO, Storme G et al. Improved survival in patients with locally advanced prostate cancer treated with radiotherapy and goserelin. $N$ Engl J Med 1997; 337: 295-300.

3 D'Amico AV, Manola J, Loffredo M, Renshaw AA, DellaCroce A, Kantoff PW. 6-month androgen suppression plus radiation therapy vs radiation therapy alone for patients with clinically localized prostate cancer: a randomized controlled trial. JAMA 2004; 292: 821-827. 
4 Alberti KG, Zimmet P, Shaw J. The metabolic syndrome--a new worldwide definition. Lancet 2005; 366: 1059-1062.

5 Mozaffarian D, Benjamin EJ, Go AS, Arnett DK, Blaha MJ, Cushman M et al. Heart disease and stroke statistics--2015 update: a report from the American Heart Association. Circulation 2015; 131: e29-322.

6 Braga-Basaria M, Dobs AS, Muller DC, Carducci MA, John M, Egan J et al. Metabolic syndrome in men with prostate cancer undergoing long-term androgen-deprivation therapy. J Clin Oncol 2006; 24: 3979-3983.

7 Stattin P, Rinaldi S, Biessy C, Stenman UH, Hallmans G, Kaaks R. High levels of circulating insulin-like growth factor-I increase prostate cancer risk: a prospective study in a population-based nonscreened cohort. J Clin Oncol 2004; 22: 3104-3112.

8 Chan JM, Stampfer MJ, Giovannucci E, Gann PH, Ma J, Wilkinson P et al. Plasma insulin-like growth factor-I and prostate cancer risk: a prospective study. Science 1998; 279: 563-566.

9 Hawley JA. Exercise as a therapeutic intervention for the prevention and treatment of insulin resistance. Diabetes Metab Res Rev 2004; 20: 383-393.

10 Pattyn N, Cornelissen VA, Eshghi SR, Vanhees L. The effect of exercise on the cardiovascular risk factors constituting the metabolic syndrome: a meta-analysis of controlled trials. Sports Med 2013; 43: 121-133.

11 Roberts CK, Hevener AL, Barnard RJ. Metabolic syndrome and insulin resistance: underlying causes and modification by exercise training. Compr Physiol 2013; 3: $1-58$.

12 Eckel RH, Grundy SM, Zimmet PZ. The metabolic syndrome. Lancet 2005; 365: 1415-1428.

13 Hammarsten J, Hogstedt B. Hyperinsulinaemia: a prospective risk factor for lethal clinical prostate cancer. Eur J Cancer 2005; 41: 2887-2895.

14 Fitzpatrick AL, Daling JR, Furberg CD, Kronmal RA, Weissfeld JL. Hypertension, heart rate, use of antihypertensives, and incident prostate cancer. Ann Epidemiol 2001; 11: 534-542.

15 Giovannucci E, Rimm EB, Stampfer MJ, Colditz GA, Willett WC. Height, body weight, and risk of prostate cancer. Cancer Epidemiol Biomarkers Prev 1997; 6: 557-563.

16 Bravi F, Scotti L, Bosetti C, Talamini R, Negri E, Montella M et al. Self-reported history of hypercholesterolaemia and gallstones and the risk of prostate cancer. Ann Oncol 2006; 17: 1014-1017.

17 Pollak M. Insulin, insulin-like growth factors and neoplasia. Best Pract Res Clin Endocrinol Metab 2008; 22: 625-638.

18 LeRoith D, Roberts CT Jr. The insulin-like growth factor system and cancer. Cancer Lett 2003; 195: 127-137.

$19 \mathrm{Yu} \mathrm{H}$, Rohan T. Role of the insulin-like growth factor family in cancer development and progression. J Natl Cancer Inst 2000; 92: 1472-1489.

20 Braun S, Bitton-Worms K, LeRoith D. The link between the metabolic syndrome and cancer. Int J Biol Sci 2011; 7: 1003-1015.

21 Gennigens C, Menetrier-Caux C, Droz JP. Insulin-Like Growth Factor (IGF) family and prostate cancer. Crit Rev Oncol Hematol 2006; 58: 124-145.

22 Gunter JH, Lubik AA, McKenzie I, Pollak M, Nelson CC. The interactions between insulin and androgens in progression to castrate-resistant prostate cancer. Adv Urol 2012; 2012: 248607.

23 Kurmasheva RT, Houghton PJ. IGF-I mediated survival pathways in normal and malignant cells. Biochim Biophys Acta 2006; 1766: 1-22.

24 Courtney KD, Corcoran RB, Engelman JA. The PI3K pathway as drug target in human cancer. J Clin Oncol 2010; 28: 1075-1083.

25 Buschemeyer WC 3rd, Freedland SJ. Obesity and prostate cancer: epidemiology and clinical implications. Eur Urol 2007; 52: 331-343.

26 Freedland SJ. Obesity and prostate cancer: a growing problem. Clin Cancer Res 2005; 11: 6763-6766.

27 De Marzo AM, Platz EA, Sutcliffe S, Xu J, Gronberg H, Drake CG et al. Inflammation in prostate carcinogenesis. Nat Rev Cancer 2007; 7: 256-269.

28 Li H, Stampfer MJ, Mucci L, Rifai N, Qiu W, Kurth T et al. A 25-year prospective study of plasma adiponectin and leptin concentrations and prostate cancer risk and survival. Clin Chem 2010; 56: 34-43.

29 Abrahamsson PA. Potential benefits of intermittent androgen suppression therapy in the treatment of prostate cancer: a systematic review of the literature. Eur Urol 2010; 57: 49-59.

30 Engel J, Bastian PJ, Baur H, Beer V, Chaussy C, Gschwend JE et al. Survival benefit of radical prostatectomy in lymph node-positive patients with prostate cancer. Eur Urol 2010; 57: 754-761.

31 Roach M 3rd, Bae K, Speight J, Wolkov HB, Rubin P, Lee RJ et al. Short-term neoadjuvant androgen deprivation therapy and external-beam radiotherapy for locally advanced prostate cancer: long-term results of RTOG 8610. J Clin Oncol 2008; 26: 585-591.

32 Studer UE, Collette L, Whelan P, Albrecht W, Casselman J, de Reijke T et al. Using PSA to guide timing of androgen deprivation in patients with T0-4 N0-2 MO prostate cancer not suitable for local curative treatment (EORTC 30891). Eur Urol 2008; 53: 941-949.
33 Horwich A, Hugosson J, de Reijke T, Wiegel T, Fizazi K, Kataja V. Prostate cancer: ESMO Consensus Conference Guidelines 2012. Ann Oncol 2013; 24: 1141-1162.

34 Heidenreich A, Bastian PJ, Bellmunt J, Bolla M, Joniau S, van der Kwast $T$ et al. EAU guidelines on prostate cancer. Part II: treatment of advanced, relapsing, and castration-resistant prostate cancer. Eur Urol 2014; 65: 467-479.

35 Garcia JA, Rini BI. Castration-resistant prostate cancer: many treatments, many options, many challenges ahead. Cancer 2012; 118: 2583-2593.

36 Keating NL, O'Malley AJ, Smith MR. Diabetes and cardiovascular disease during androgen deprivation therapy for prostate cancer. J Clin Oncol 2006; 24: 4448-4456.

37 Keating NL, O'Malley AJ, Freedland SJ, Smith MR. Diabetes and cardiovascular disease during androgen deprivation therapy: observational study of veterans with prostate cancer. J Natl Cancer Inst 2010; 102: 39-46.

38 Van Hemelrijck M, Garmo H, Holmberg L, Ingelsson E, Bratt O, Bill-Axelson A et al. Absolute and relative risk of cardiovascular disease in men with prostate cancer: results from the Population-Based PCBaSe Sweden. J Clin Oncol 2010; 28: 3448-3456.

39 Taylor LG, Canfield SE, Du XL. Review of major adverse effects of androgendeprivation therapy in men with prostate cancer. Cancer 2009; 115: 2388-2399.

40 Smith MR, Finkelstein JS, McGovern FJ, Zietman AL, Fallon MA, Schoenfeld DA et al. Changes in body composition during androgen deprivation therapy for prostate cancer. J Clin Endocrinol Metab 2002; 87: 599-603.

41 Haseen F, Murray LJ, Cardwell CR, O'Sullivan JM, Cantwell MM. The effect of androgen deprivation therapy on body composition in men with prostate cancer: systematic review and meta-analysis. J Cancer Surviv 2010; 4: 128-139.

42 Boxer RS, Kenny AM, Dowsett R, Taxel P. The effect of 6 months of androgen deprivation therapy on muscle and fat mass in older men with localized prostate cancer. Aging Male 2005; 8: 207-212.

43 Storer TW, Miciek R, Travison TG. Muscle function, physical performance and body composition changes in men with prostate cancer undergoing androgen deprivation therapy. Asian J Androl 2012; 14: 204-221.

44 Dockery F, Bulpitt CJ, Agarwal S, Donaldson M, Rajkumar C. Testosterone suppression in men with prostate cancer leads to an increase in arterial stiffness and hyperinsulinaemia. Clin Sci (Lond) 2003; 104: 195-201.

45 Eri LM, Urdal P, Bechensteen AG. Effects of the luteinizing hormone-releasing hormone agonist leuprolide on lipoproteins, fibrinogen and plasminogen activator inhibitor in patients with benign prostatic hyperplasia. J Urol 1995; 154: 100-104.

46 Basaria S, Muller DC, Carducci MA, Egan J, Dobs AS. Hyperglycemia and insulin resistance in men with prostate carcinoma who receive androgen-deprivation therapy. Cancer 2006; 106: 581-588.

47 Smith JC, Bennett S, Evans LM, Kynaston HG, Parmar M, Mason MD et al. The effects of induced hypogonadism on arterial stiffness, body composition, and metabolic parameters in males with prostate cancer. $J$ Clin Endocrinol Metab 2001; 86: 4261-4267.

48 Smith MR, Lee H, Fallon MA, Nathan DM. Adipocytokines, obesity, and insulin resistance during combined androgen blockade for prostate cancer. Urology 2008; 71: 318-322.

49 Smith MR, Lee $\mathrm{H}$, Nathan DM. Insulin sensitivity during combined androgen blockade for prostate cancer. J Clin Endocrinol Metab 2006; 91: 1305-1308.

50 Rubinow KB, Snyder CN, Amory JK, Hoofnagle AN, Page ST. Acute testosterone deprivation reduces insulin sensitivity in men. Clin Endocrinol (Oxf) 2012; 76: 281-288.

51 Page ST, Herbst KL, Amory JK, Coviello AD, Anawalt BD, Matsumoto AM et al. Testosterone administration suppresses adiponectin levels in men. J Androl 2005; 26: 85-92.

52 Ketchandji M, Kuo YF, Shahinian VB, Goodwin JS. Cause of death in older men after the diagnosis of prostate cancer. J Am Geriatr Soc 2009; 57: 24-30.

53 Levine GN, D'Amico AV, Berger P, Clark PE, Eckel RH, Keating NL et al. Androgendeprivation therapy in prostate cancer and cardiovascular risk: a science advisory from the American Heart Association, American Cancer Society, and American Urological Association: endorsed by the American Society for Radiation Oncology. Circulation 2010; 121: 833-840.

54 Allan CA, Collins VR, Frydenberg M, McLachlan Rl, Matthiesson KL. Androgen deprivation therapy complications. Endocr Relat Cancer 2014; 21: T119-T129.

55 Conteduca V, Di Lorenzo G, Bozza G, Ardito R, Aieta M. Metabolic syndrome as a peculiar target for management of prostate cancer patients. Clin Genitourin Cancer 2013; 11: 211-220.

56 Balducci S, Zanuso S, Nicolucci A, Fernando F, Cavallo S, Cardelli P et al. Antiinflammatory effect of exercise training in subjects with type 2 diabetes and the metabolic syndrome is dependent on exercise modalities and independent of weight loss. Nutr Metab Cardiovasc Dis 2010; 20: 608-617.

57 Tjonna AE, Lee SJ, Rognmo O, Stolen TO, Bye A, Haram PM et al. Aerobic interval training versus continuous moderate exercise as a treatment for the metabolic syndrome: a pilot study. Circulation 2008; 118: 346-354. 
58 Stewart KJ, Bacher AC, Turner K, Lim JG, Hees PS, Shapiro EP et al. Exercise and risk factors associated with metabolic syndrome in older adults. Am J Prev Med 2005; 28: 9-18.

59 Johnson JL, Slentz CA, Houmard JA, Samsa GP, Duscha BD, Aiken LB et al. Exercise training amount and intensity effects on metabolic syndrome (from Studies of a Targeted Risk reduction Intervention Through Defined Exercise). Am J Cardiol 2007; 100: 1759-1766.

60 Katzmarzyk PT, Leon AS, Wilmore JH, Skinner JS, Rao DC, Rankinen T et al. Targeting the metabolic syndrome with exercise: evidence from the HERITAGE Family Study. Med Sci Sports Exerc 2003; 35: 1703-1709.

61 Bateman LA, Slentz CA, Willis LH, Shields AT, Piner LW, Bales CW et al. Comparison of aerobic versus resistance exercise training effects on metabolic syndrome (from the Studies of a Targeted Risk Reduction Intervention Through Defined Exercise - STRRIDE-AT/RT). Am J Cardiol 2011; 108: 838-844

62 Stensvold D, Tjonna AE, Skaug EA, Aspenes S, Stolen T, Wisloff U et al. Strength training versus aerobic interval training to modify risk factors of metabolic syndrome. J Appl Physiol (1985) 2010; 108: 804-810.

63 Hwang CL, Wu YT, Chou CH. Effect of aerobic interval training on exercise capacity and metabolic risk factors in people with cardiometabolic disorders: a meta-analysis. J Cardiopulm Rehabil Prev 2011; 31: 378-385.

64 Gibala MJ, Little JP, Macdonald MJ, Hawley JA. Physiological adaptations to low-volume, high-intensity interval training in health and disease. J Physiol 2012; 590: 1077-1084.

65 Bourke L, Doll H, Crank H, Daley A, Rosario D, Saxton JM. Lifestyle intervention in men with advanced prostate cancer receiving androgen suppression therapy: a feasibility study. Cancer Epidemiol Biomarkers Prev 2011; 20: 647-657.

66 Bourke L, Gilbert S, Hooper R, Steed LA, Joshi M, Catto JW et al. Lifestyle changes for improving disease-specific quality of life in sedentary men on long-term androgen-deprivation therapy for advanced prostate cancer: a randomised controlled trial. Eur Urol 2014; 65: 865-872.

67 Cormie P, Galvao DA, Spry N, Joseph D, Chee R, Taaffe DR et al. Can supervised exercise prevent treatment toxicity in patients with prostate cancer initiating androgen-deprivation therapy: a randomised controlled trial. BJU Int 2015; 115: 256-266.

68 Culos-Reed SN, Robinson JW, Lau H, Stephenson L, Keats M, Norris S et al. Physical activity for men receiving androgen deprivation therapy for prostate cancer: benefits from a 16-week intervention. Support Care Cancer 2010; 18: 591-599.

69 Galvao DA, Taaffe DR, Spry N, Joseph D, Newton RU. Combined resistance and aerobic exercise program reverses muscle loss in men undergoing androgen suppression therapy for prostate cancer without bone metastases: a randomized controlled trial. J Clin Oncol 2010; 28: 340-347.

70 Hvid T, Winding K, Rinnov A, Dejgaard T, Thomsen C, Iversen P et al. Endurance training improves insulin sensitivity and body composition in prostate cancer patients treated with androgen deprivation therapy. Endocr Relat Cancer 2013; 20: 621-632.

71 Santa Mina D, Connor MK, Alibhai SM, Toren P, Guglietti C, Matthew AG et al. Exercise effects on adipokines and the IGF axis in men with prostate cancer treated with androgen deprivation: a randomized study. Can Urol Assoc J 2013; 7: E692-E698.

72 Segal RJ, Reid RD, Courneya KS, Malone SC, Parliament MB, Scott CG et al. Resistance exercise in men receiving androgen deprivation therapy for prostate cancer. J Clin Oncol 2003; 21: 1653-1659.

73 Kushi LH, Doyle C, McCullough M, Rock CL, Demark-Wahnefried W, Bandera EV et al. American Cancer Society Guidelines on nutrition and physical activity for cancer prevention: reducing the risk of cancer with healthy food choices and physical activity. CA Cancer J Clin 2012; 62: 30-67.

74 National Cancer Institute UoSC. Resistance Training and Protein Supplementation in Increasing Lean Body Mass for Prostate Cancer Survivors Receiving Androgen Deprivation Therapy: Bethesda, MD, USA 2016.

75 Haff GG. Roundtable discussion: periodization of training-part 1. Strength Cond $J$ 2004; 26: 50-69.

76 Rigshospitalet D. Effectiveness of Community-based Football in Prostate Cancer (FC-PC) 2016

77 Vest H. Xbox Kinect Training in Men With Prostate Cancer 2016.

78 Hospital H. Supervised Group Based Exercise for Men With Prostate Cancer on Androgen Deprivation Therapy 2016.

79 University. NCIG. An Exercise Intervention to Mitigate Side-Effects Related to Androgen Deprivation Therapy Among Prostate Cancer Survivors. National Library of Medicine (US): Bethesda, MD, USA, 2016

80 Gleeson M, Bishop NC, Stensel DJ, Lindley MR, Mastana SS, Nimmo MA. The anti-inflammatory effects of exercise: mechanisms and implications for the prevention and treatment of disease. Nat Rev Immunol 2011; 11: 607-615.
81 Samuel VT, Shulman GI. Mechanisms for insulin resistance: common threads and missing links. Cell 2012; 148: 852-871.

82 Pedersen BK, Febbraio MA. Muscles, exercise and obesity: skeletal muscle as a secretory organ. Nat Rev Endocrinol 2012; 8: 457-465.

83 Christ-Roberts CY, Pratipanawatr T, Pratipanawatr W, Berria R, Belfort R, Kashyap $S$ et al. Exercise training increases glycogen synthase activity and GLUT4 expression but not insulin signaling in overweight nondiabetic and type 2 diabetic subjects. Metabolism 2004; 53: 1233-1242.

84 Hughes VA, Fiatarone MA, Fielding RA, Kahn BB, Ferrara CM, Shepherd P et al. Exercise increases muscle GLUT-4 levels and insulin action in subjects with impaired glucose tolerance. Am J Physiol 1993; 264: E855-E862.

85 Little JP, Gillen JB, Percival ME, Safdar A, Tarnopolsky MA, Punthakee Z et al. Low-volume high-intensity interval training reduces hyperglycemia and increases muscle mitochondrial capacity in patients with type 2 diabetes. $J$ Appl Physiol (1985) 2011; 111: 1554-1560.

86 Wende AR, Schaeffer PJ, Parker GJ, Zechner C, Han DH, Chen MM et al. A role for the transcriptional coactivator PGC-1alpha in muscle refueling. J Biol Chem 2007; 282: 36642-36651.

87 Stocks T, Rapp K, Bjorge T, Manjer J, Ulmer H, Selmer R et al. Blood glucose and risk of incident and fatal cancer in the metabolic syndrome and cancer project (me-can): analysis of six prospective cohorts. PLoS Med 2009; 6: e1000201.

88 D'Amico AV, Braccioforte MH, Moran BJ, Chen MH. Causes of death in men with prevalent diabetes and newly diagnosed high- versus favorable-risk prostate cancer. Int J Radiat Oncol Biol Phys 2010; 77: 1329-1337.

89 Turcotte LP, Fisher JS. Skeletal muscle insulin resistance: roles of fatty acid metabolism and exercise. Phys Ther 2008; 88: 1279-1296.

90 Shojaee-Moradie F, Baynes KC, Pentecost C, Bell JD, Thomas EL, Jackson NC et al. Exercise training reduces fatty acid availability and improves the insulin sensitivity of glucose metabolism. Diabetologia 2007; 50: 404-413.

91 van Aggel-Leijssen DP, Saris WH, Wagenmakers AJ, Senden JM, van Baak MA. Effect of exercise training at different intensities on fat metabolism of obese men. J Appl Physiol (1985) 2002; 92: 1300-1309.

92 Menshikova EV, Ritov VB, Toledo FG, Ferrell RE, Goodpaster BH, Kelley DE. Effects of weight loss and physical activity on skeletal muscle mitochondrial function in obesity. Am J Physiol Endocrinol Metab 2005; 288: E818-E825.

93 Kobayashi N, Barnard RJ, Said J, Hong-Gonzalez J, Corman DM, Ku M et al. Effect of low-fat diet on development of prostate cancer and Akt phosphorylation in the Hi-Myc transgenic mouse model. Cancer Res 2008; 68: 3066-3073.

94 Pettersson A, Lis RT, Meisner A, Flavin R, Stack EC, Fiorentino M et al. Modification of the association between obesity and lethal prostate cancer by TMPRSS2:ERG. J Natl Cancer Inst 2013; 105: 1881-1890.

95 Schlaepfer IR, Rider L, Rodrigues LU, Gijon MA, Pac CT, Romero L et al. Lipid catabolism via CPT1 as a therapeutic target for prostate cancer. Mol Cancer Ther 2014; 13: 2361-2371

96 Ouchi N, Parker JL, Lugus JJ, Walsh K. Adipokines in inflammation and metabolic disease. Nat Rev Immunol 2011; 11: 85-97.

97 Wekesa A, Harrison M, Watson RW. Physical activity and its mechanistic effects on prostate cancer. Prostate Cancer Prostatic Dis 2015; 18: 197-207.

98 Fischer CP. Interleukin-6 in acute exercise and training: what is the biological relevance. Exerc Immunol Rev 2006; 12: 41.

99 Keller C, Steensberg A, Hansen AK, Fischer CP, Plomgaard P, Pedersen BK. Effect of exercise, training, and glycogen availability on IL-6 receptor expression in human skeletal muscle. J Appl Physiol 2005; 99: 2075-2079.

100 Carey AL, Steinberg GR, Macaulay SL, Thomas WG, Holmes AG, Ramm G et al. Interleukin- 6 increases insulin-stimulated glucose disposal in humans and glucose uptake and fatty acid oxidation in vitro via AMP-activated protein kinase. Diabetes 2006; 55: 2688-2697.

101 Kim H-J, Higashimori T, Park S-Y, Choi H, Dong J, Kim Y-J et al. Differential effects of interleukin- 6 and-10 on skeletal muscle and liver insulin action in vivo. Diabetes 2004; 53: 1060-1067.

102 Pedersen L, Idorn M, Olofsson GH, Lauenborg B, Nookaew I, Hansen RH et al. Voluntary running suppresses tumor growth through epinephrine-and IL-6dependent NK cell mobilization and redistribution. Cell Metab 2016; 23: 554-562.

103 Jones LW, Antonelli J, Masko EM, Broadwater G, Lascola CD, Fels D et al. Exercise modulation of the host-tumor interaction in an orthotopic model of murine prostate cancer. J Appl Physiol (1985) 2012; 113: 263-272.

104 Nakhla AM, Rosner W. Stimulation of prostate cancer growth by androgens and estrogens through the intermediacy of sex hormone-binding globulin. Endocrinology 1996; 137: 4126-4129.

105 Rundqvist $\mathrm{H}$, Augsten M, Stromberg A, Rullman E, Mijwel S, Kharaziha $\mathrm{P}$ et al. Effect of acute exercise on prostate cancer cell growth. PLoS One 2013; 8: e67579.

106 Tymchuk CN, Barnard RJ, Heber D, Aronson WJ. Evidence of an inhibitory effect of diet and exercise on prostate cancer cell growth. J Urol 2001; 166: 1185-1189. 
107 Barnard RJ, Ngo TH, Leung PS, Aronson WJ, Golding LA. A low-fat diet and/or strenuous exercise alters the IGF axis in vivo and reduces prostate tumor cell growth in vitro. Prostate 2003; 56: 201-206.

108 Barnard RJ, Leung PS, Aronson WJ, Cohen P, Golding LA. A mechanism to explain how regular exercise might reduce the risk for clinical prostate cancer Eur J Cancer Prev 2007; 16: 415-421.

109 Ngo TH, Barnard RJ, Leung PS, Cohen P, Aronson WJ. Insulin-like growth factor I (IGF-I) and IGF binding protein-1 modulate prostate cancer cell growth and apoptosis: possible mediators for the effects of diet and exercise on cancer cell survival. Endocrinology 2003; 144: 2319-2324.
This work is licensed under a Creative Commons Attribution 4.0 International License. The images or other third party material in this article are included in the article's Creative Commons license, unless indicated otherwise in the credit line; if the material is not included under the Creative Commons license, users will need to obtain permission from the license holder to reproduce the material. To view a copy of this license, visit http://creativecommons.org/licenses/ by/4.0/

(c) The Author(s) 2016 